\title{
Supercurrent-carrying density of states in diffusive mesoscopic Josephson weak links
}

\author{
Tero T. Heikkilä, ${ }^{1,2, *}$ Jani Särkkä, ${ }^{1}$ and Frank K. Wilhelm ${ }^{3}$ \\ ${ }^{1}$ Materials Physics Laboratory, Helsinki University of Technology, P.O. Box 2200, FIN-02015 HUT, Finland \\ ${ }^{2}$ Institut für Theoretische Festkörperphysik, Universität Karlsruhe, D-76128 Karlsruhe, Germany \\ ${ }^{3}$ Sektion Physik and CeNS, Ludwig-Maximilians-Universität, Theresienstr. 37, D-80333 München, Germany
}

(Dated: October 27, 2018)

\begin{abstract}
Recent experiments have demonstrated the nonequilibrium control of the supercurrent through diffusive phase-coherent normal-metal weak links. The experimental results have been accurately described by the quasiclassical Green's function technique in the Keldysh formalism. Taking into account the geometry of the structure, different energy scales and the nonidealities at the interfaces allows us to obtain a quantitative agreement between the theory and the experimental results in both the amplitude and the phase dependence of the supercurrent, with no or very few fitting parameters. Here we discuss the most important factors involved with such comparisons: the ratio between the superconducting order parameter and the Thouless energy of the junction, the effect of additional wires on the weak link, and the effects due to imperfections, most notably due to the nonideal interfaces.
\end{abstract}

PACS numbers: 74.50.+r, 73.23.-b, 74.40.+k, 74.80.Fp

\section{INTRODUCTION}

Many quantum phenomena in many-body systems are based on probing the spectrum of states corresponding to the desired observable, the states being filled according to an appropriate distribution function. A similar viewpoint can be taken also on the Josephson effect: supercurrent is carried by states in the weak link and their occupation is determined by a distribution function antisymmetric between the electron and hole spaces. This aspect is directly reflected in the mathematical structure of the supercurrent formula derived from the Keldysh Green'sfunctions method. ${ }^{1-3}$ Such an approach has been taken in some recent experiments ${ }^{4-9}$ controlling the Josephson effect in phase-coherent normal-metal wires through the control of the distribution function by an injection of normal quasiparticle current. One of the most remarkable results of these experiments is the inversion of the sign of the supercurrent for a given phase difference across the weak link when the junctions turn into a $\pi$ state.

Quantitative fit to the experimentally obtained results has been very successful for the equilibrium supercurrent ${ }^{10}$ using the equilibrium quasiclassical theory. In the nonequilibrium case, detailed knowledge of the relaxation mechanisms controlling the shape of the interactions, but also the precise spectrum of supercurrent-carrying states, is required. ${ }^{6,8}$ Previously, ${ }^{1}$ for the calculation of this spectrum, one has assumed a two-probe setup with some idealized conditions on the length scales and on the nature of the interfaces. In this paper, we systematically investigate the spectrum of this current-carrying density of states, or spectral supercurrent, show how it is calculated, and how it depends on the length of the weak link, presence of additional terminals, or on the nonidealities in the interfaces between the normal-metal weak link and the superconductors. We also discuss the current-phase relation of such a system: at low temperatures, it can be far from sinusoidal, and at certain conditions, its period can even be halved. ${ }^{11} \mathrm{We}$ focus on the diffusive limit where the dimensions $d$ of the weak link are much greater than the elastic mean free path $l$. This is the typical limit for most normal-metal weak links. The corresponding ballistic limit $d \ll l$ has been extensively described in the literature ${ }^{12-18}$ in terms of Andreev bound states (ABS). We show qualitatively a connection between the discrete ABS and the continuous diffusive-limit spectral supercurrent.

This paper is organized as follows. After this introduction, Sec. II introduces to the theoretical formalism which is based on the real-time Usadel equation for the quasiclassical Green's function in the diffusive limit. ${ }^{19,20}$ In the case of nonideal interfaces or in multiterminal geometries, the boundary conditions to these functions are also essential. Understanding the results of the following sections does not require a detailed reading of this part but it is enough to grasp the idea of the relation of the spectral supercurrent and the observable one. In Sec. III we look how the spectral supercurrent depends on the length of the weak link compared to the superconducting coherence length and separate two extreme cases. In the limit of a short junction where the coherence length is much longer than the weak link, one obtains an analytical solution for the spectral supercurrent without further approximations. The current-phase relation in diffusive normal-metal weak links is considered in Sec. IV. We show how, especially at low temperatures, higher harmonics appear in addition to the usual sinusoidal phase dependence and indicate how the period can be halved in a nonequilibrium situation. Section V considers the effect of additional normal-metal terminals on the current-carrying density of states, and in Sec. VI, we discuss how nonidealities in the normal metal - superconductor (NS) interface change its shape. Finally, in Sec. VII, we summarize the main results.

To be specific, we consider the structure shown in Fig. 1. The main wire with length $L$ and cross section $A_{w}$ be- 


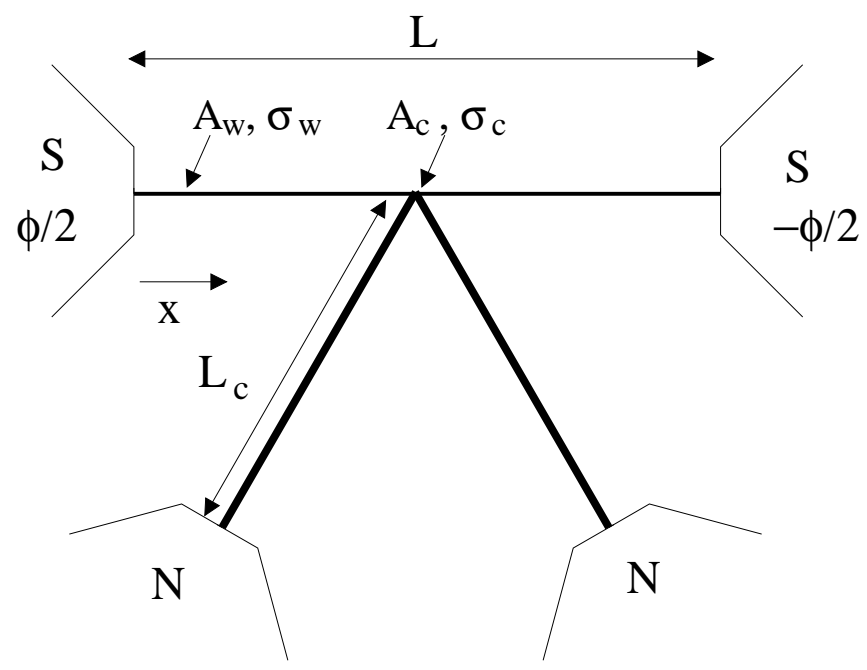

FIG. 1: Multiterminal SNS Josephson junction. The weak link consists of a phase coherent normal-metal wire of length $L$, cross section $A_{w}$, and normal-state conductivity $\sigma_{w}$, yielding a normal-state resistance $R_{N}=L / \sigma_{w} A_{w}$. Additional normal-metal wires of length $L_{c}$, total cross section $A_{c}$, and normal-state conductivity $\sigma_{c}$, i.e., with resistance $R_{c}=$ $L_{c} / \sigma_{w} A_{w}$, called the control wires, are connected to the center of the weak link, and from their other end, to normal reservoirs.

tween the superconductors forms the weak link whereas the additional wires with length $L_{c}$ and integrated cross section $A_{c}$ are used for the control of the distribution functions and therefore referred to as the control wires. We assume that the superconducting and normal reservoirs are much larger than the weak link and the control wire, such that the Green's functions describing them take their bulk values very close to the interfaces. Furthermore, we assume that the width of the control wires is much smaller than the length $L$ of the weak links. This allows us to consider the wires as quasi-one-dimensional structures by assuming translational invariance in the transverse directions.

\section{THEORETICAL BACKGROUND}

Circuits composed of normal and superconducting metals in the diffusive limit (dimensions larger than the elastic mean free path $l$ ) are effectively described in terms of the quasiclassical Green's functions $\breve{G}$ satisfying the Usadel equations ${ }^{19,20}$

$$
D \nabla(\breve{G} \nabla \breve{G})=\left[-i(E+i \Gamma) \breve{\tau_{3}}+\breve{\Delta}, \breve{G}\right]
$$

where $D=\frac{1}{3} v_{F} l$ is the diffusion constant, $E$ is energy relative to the chemical potential of the superconductors (which is assumed to be the same for all $\mathrm{S}$ terminals), $\Gamma$ describes a small inelastic scattering rate, and $\breve{\Delta}$ the superconducting pair potential (we set $\hbar=1$ throughout). Since we aim to describe nonequilibrium effects, we adopt the Keldysh real-time formalism ${ }^{21}$ and hence

$$
\breve{G}=\left(\begin{array}{cc}
\hat{G}^{R} & \hat{G}^{K} \\
0 & \hat{G}^{A}
\end{array}\right), \quad \breve{\Delta}=\left(\begin{array}{cc}
\hat{\Delta} & 0 \\
0 & \hat{\Delta}
\end{array}\right), \quad \breve{\tau}_{3}=\left(\begin{array}{cc}
\hat{\tau}_{3} & 0 \\
0 & \hat{\tau}_{3}
\end{array}\right)
$$

All of the submatrices denoted by a hat $\left(\hat{G}^{R}\right.$, etc. $)$ are $2 \times 2$ matrices in Nambu particle-hole space, in particular, $\hat{\tau}_{3}$ is the third Pauli matrix and $\hat{\Delta}$ has the form

$$
\hat{\Delta}=\left(\begin{array}{cc}
0 & \Delta(x) \\
\Delta^{*}(x) & 0
\end{array}\right)
$$

The pair potential $\Delta(x)$ can in principle be obtained from a self-consistency relation. ${ }^{20,22}$ However, since we consider only superconducting reservoirs much wider than the weak link, we adopt the usual step-function form for $\Delta(x)$ (finite constant in the superconductors, zero in the normal-metal wires).

In addition to Eq. (1), Usadel Green's function satisfies a normalization condition $\breve{G}^{2}=\check{1}$. Therefore it can be parametrized with four scalar parameters as follows. ${ }^{20}$ The Keldysh Green's function $\hat{G}^{K}$ describing the occupation numbers of different quantum states, i.e., the (non)equilibrium state of the system can be expressed with two real distribution functions $f_{L}$ and $f_{T}$ as $\hat{G}^{K}=\hat{G}^{R}\left(f_{L}+f_{T} \hat{\tau}_{3}\right)-\left(f_{L}+f_{T} \hat{\tau}_{3}\right) \hat{G}^{A}$ whereas the retarded and advanced Green's functions, $\hat{G}^{R}$ and $\hat{G}^{A}$, describing the spectral properties which do not directly depend on the distribution functions are

$$
\hat{G}^{R}=\left(\begin{array}{cc}
\cosh (\theta) & \sinh (\theta) \exp (i \chi) \\
-\sinh (\theta) \exp (-i \chi) & -\cosh (\theta)
\end{array}\right)
$$

and $\hat{G}^{A}=-\hat{\tau}_{3}\left(\hat{G}^{R}\right)^{\dagger} \hat{\tau}_{3}$. Here $\theta(x ; E)$ and $\chi(x ; E)$ are in general complex scalar functions.

In what follows, we describe a quasi-one-dimensional situation, where the functions are assumed to vary only in one dimension $x$. Expressing the coordinate $x$ in terms of the separation $L$ of the superconductors between which the supercurrent flows, $x \equiv x^{\prime} L$, the spectral equations for $\hat{G}^{R(A)}$ read in a normal metal $(\Delta=0)$

$$
\begin{aligned}
\partial_{x^{\prime}}^{2} \theta & =-2 i\left(E^{\prime}+i \Gamma^{\prime}\right) \sinh (\theta)+\frac{1}{2}\left(\partial_{x^{\prime}} \chi\right)^{2} \sinh (2 \theta) \\
j_{E} & \equiv-\sinh ^{2}(\theta) \partial_{x^{\prime}} \chi, \quad \partial_{x^{\prime}} j_{E}=0 .
\end{aligned}
$$

Here, the prime over the (dimensionless) quantities denotes the fact that the energies are expressed in the units of the Thouless energy $E_{T}=D / L^{2}$ corresponding to the length $L$. Below, we tacitly assume all lengths and energies expressed in these natural units even if not marked by a prime. The kinetic equations satisfied by the distribution functions $f_{L}$ and $f_{T}$ are described, e.g., in Ref. 20 , where the part of the distribution function which is symmetric about the chemical potential of the superconductors corresponds to $f_{T}$ and the antisymmetric part to $f_{L}$. These two components acquire different space and energy dependent diffusion coefficients due to the superconducting proximity effect. 
If the interfaces to the reservoirs are ideal metallic, the parameters are continuous at the boundaries to the reservoirs and can be identified with the bulk values, $\theta_{S}=\operatorname{artanh}(\Delta / E)$ and $\theta_{N}=0$ in the superconducting and normal-metal reservoirs, respectively. In general, e.g., if a supercurrent is driven through the system, there can be a phase difference, which we choose to be applied symmetrically between the superconductors, such that in the left superconductor $\chi=\phi / 2$ and in the right $\chi=-\phi / 2$. Below, if not mentioned otherwise, we choose $\phi=\pi / 2$, which typically yields a supercurrent close to the critical current of the junction.

Nonideal interfaces with reduced transmissivities are not directly described by the Usadel equation, because they are of microscopic, atomic-scale thickness. They can, however, be taken into account using boundary conditions derived by Zaitsev ${ }^{23}$ for Eilenberger Green's functions (valid independent of the mean free path) and later simplified in the diffusive limit by Kuprianov and Lukichev for a tunneling case ${ }^{24}$ and Nazarov for a general interface, ${ }^{25}$ described by a scattering matrix. For an interface characterized by the transmission eigenvalues $T_{n}$, the Green's functions $\breve{G}_{1}$ on the right-hand side and $G_{2}$ on the left-hand side of the interface satisfy ${ }^{25,26}$

$$
\begin{aligned}
\sigma_{N}^{1} A_{1} \breve{G}_{1} \partial_{x} \breve{G}_{1} & =\sigma_{N}^{2} A_{2} \breve{G}_{2} \partial_{x} \breve{G}_{2} \\
& =\frac{2 e^{2}}{\pi} \sum_{n} \frac{T_{n}\left[\breve{G}_{1}, \breve{G}_{2}\right]}{4+T_{n}\left(\left\{\breve{G}_{1}, \breve{G}_{2}\right\}-2\right)},
\end{aligned}
$$

evaluated at the position of the interface. In most cases, the individual transmission eigenvalues are not known, but since typical interfaces contain a huge number of channels, it is enough to integrate over the probability distribution of the eigenvalues to obtain the desired boundary condition.

In the case of a tunneling interface (where all the transmission eigenvalues of the interface are small) the boundary conditions between the parametrized functions in wires 1 and 2 reduce to ${ }^{20,24}$

$$
\partial_{x} \theta_{1}=\left[\sinh \left(\theta_{1}\right) \cosh \left(\theta_{2}\right)-\sinh \left(\theta_{2}\right) \cosh \left(\theta_{1}\right) \cos (\Delta \chi)\right] / r_{b},
$$

$$
\sinh ^{2}\left(\theta_{1}\right) \partial_{x} \chi_{1}=\sinh \left(\theta_{1}\right) \sinh \left(\theta_{2}\right) \sin (\Delta \chi) / r_{b}
$$

Here, $\Delta \chi \equiv \chi_{1}-\chi_{2}$ and $\theta_{1(2)} \equiv \theta\left(x_{b}^{+(-)}\right)$and $\chi_{1(2)} \equiv$ $\chi\left(x_{b}^{+(-)}\right)$are the parameters $\theta$ and $\chi$ at the interface, $x=x_{b}$, but on the side of the wire 1 (2). The nonideality of the interface is characterized by the ratio of its resistance $R_{I}$ and of the weak-link resistance $R_{N}$, $r_{b} \equiv R_{I} / R_{N}$ and the derivatives point towards the wire 1. In the case of a dirty interface, where the boundary condition is evaluated using the distribution function of the transmission eigenvalues corresponding to an interface with a random array of scatterers in a $2 \mathrm{D}$ layer, ${ }^{27}$ we get

$$
\partial_{x} \theta_{1}=\frac{\sqrt{2}\left[\sinh \left(\theta_{1}\right) \cosh \left(\theta_{2}\right)-\sinh \left(\theta_{2}\right) \cosh \left(\theta_{1}\right) \cos (\Delta \chi)\right]}{r_{b} \mathcal{D}},
$$

$\sinh ^{2}\left(\theta_{1}\right) \partial_{x} \chi_{1}=\frac{\sqrt{2} \sinh \left(\theta_{2}\right) \sinh \left(\theta_{1}\right) \sin (\Delta \chi)}{r_{b} \mathcal{D}}$.

Here we denoted the denominator $\mathcal{D} \equiv$ $\sqrt{1+\cosh \left(\theta_{2}\right) \cosh \left(\theta_{1}\right)-\sinh \left(\theta_{2}\right) \sinh \left(\theta_{1}\right) \cos (\Delta \chi)}$.

This denominator reflects the contribution of open conduction channels which are not present in Eq. (9).

Note that both types of boundary conditions indicate a form of a conservation of a spectral current over the interface, the second equation being the conservation of the spectral supercurrent $j_{E}$.

In geometries with more than two terminals, we assume that narrow quasi-one-dimensional wires connect to each other at some point of the structure. Therefore we need to impose appropriate matching conditions..$^{20,25,28,29}$ In this case, they are the continuity of the functions $\theta$ and $\chi$ and the conservation of the spectral currents. Assuming that the derivatives in the $N$ wires $i=1, \ldots N$ with cross sections $A_{i}$ and normalstate conductivities $\sigma_{N}^{i}$ point towards the crossing point at $x_{c}$, we get

$$
\begin{aligned}
& \theta_{i}\left(x_{c}\right)=\theta_{j}\left(x_{c}\right) \quad \forall i, j=1, \ldots N, \\
& \chi_{i}\left(x_{c}\right)=\chi_{j}\left(x_{c}\right) \quad \forall i, j=1, \ldots N, \\
& \sum_{i=1}^{N} A_{i} \sigma_{N}^{i} \partial_{x} \theta_{i}\left(x_{c}\right)=0, \\
& \sum_{i=1}^{N} A_{i} \sigma_{N}^{i} \partial_{x} \chi_{i}\left(x_{c}\right)=0 .
\end{aligned}
$$

In the last condition we used the continuity of the parameters $\theta$ across the crossing point.

Below, we assume the system depicted in Fig. 1: two superconductors connected by "horizontal" mesoscopic normal wires to which we connect normal reservoirs by the "vertical" mesoscopic normal wires (labeling of the wires as in Fig. 1). When considering the supercurrent between the two superconductors, for the spectral equations it is enough to treat any number of "vertical" wires by a single wire for which the product of $\sigma_{N} A$ is simply the sum of these products in the individual wires. In the case that the dependence on the length $L_{c}$ of these wires becomes important, the smallest of them characterizes the situation the best. In this case, since there can be no supercurrent flowing to the normal reservoirs, Eq. (15) reduces to $j_{E}^{1}=-j_{E}^{2}$. Furthermore, for simplicity, we assume the system left-right symmetric, such that the part of the weak link in the left-hand side of the cross is similar to that in the right-hand side.

Finally, the observable supercurrent is obtained from the solutions to the spectral and kinetic equations by

$$
I_{S}=\frac{E_{T}}{2 e R_{N}} \int_{-\infty}^{\infty} d E^{\prime} \operatorname{Im}\left[j_{E}\left(E^{\prime}\right)\right] f_{L}\left(E^{\prime}\right) .
$$


In the reservoirs with voltage $V$ with respect to the potential of the superconductors (which are assumed equal for both superconductors in order to avoid the ac Josephson effect), $f_{L}$ obtains the form

$$
f_{L}(E ; V, T)=\frac{1}{2}\left[\tanh \left(\frac{E+e V}{2 k_{B} T}\right)+\tanh \left(\frac{E-e V}{2 k_{B} T}\right)\right] .
$$

It can be shown ${ }^{1,6}$ that, in the absence of inelastic interactions and for energies $E<\Delta, f_{L}$ remains constant throughout the control wires, and hence the reservoir value can directly be used for the calculation of the supercurrent.

In this paper, we will consider two limits for $f_{L}$. These are the equilibrium finite-temperature limit, where $f_{L}=\tanh \left(E / 2 k_{B} T\right)$, and the zero-temperature nonequilibrium case when $f_{L}$ is driven in a normal-metal wire, ${ }^{1}$ $f_{L}=\vartheta(E-e V)-\vartheta(-e V-E)$, where $\vartheta(E)$ is the Heaviside step function.

The spectrum of supercurrent-carrying states typically consists of both the states carrying the supercurrent parallel to the phase gradient and those carrying it in the opposite direction, ${ }^{1,12}$ depending on their energy. Hence, by controlling the occupation of these states by the above steplike distribution function, one is able to vary the sign of the observable supercurrent and, e.g., obtain the $\pi$ state.

The form of the spectrum can be qualitatively understood by considering a ballistic (scattering-free) weak link. There, the quasiparticles form bound states ${ }^{12-18}$ which contain an Andreev reflection ${ }^{30}$ at both NS interfaces. Since the first reflection at the left is from holeto particle (particle- to hole) -like states and the second at the right interface from particle- to hole (from a holeto particle) -like states, the net result is a transfer of a Cooper pair from the left superconductor to the right (from right to left). Bound-state energies are found by requiring that the total phase the quasiparticles acquire within a single cycle is a multiple of $2 \pi$. This leads to (for $E_{m} \ll \Delta$ )

$$
E_{m}^{ \pm}=\frac{1}{2 \tau}\left[2 \pi\left(m+\frac{1}{2}\right) \pm \phi\right]
$$

the sign in front of the phase depending on the direction of the supercurrent flow. Here $\tau=v_{F} / L$ is the time of flight between two successive Andreev reflections and $L$ is the corresponding length of the trajectory. The supercurrent-carrying density of states is then found from

$$
j_{S}(E ; \phi) \propto \sum_{m} \frac{\partial E_{m}^{ \pm}}{\partial \phi} \delta\left(E-E_{m}\right)
$$

resulting into a peaklike spectrum that contains states carrying both positive $\left(E=E_{m}^{+}\right)$and negative $\left(E=E_{m}^{-}\right)$ supercurrent. In the presence of disorder, the distribution of the times of flight $\tau$ depends on the impurity potential and the spectral supercurrent is conveniently characterized by its impurity-averaged smooth density of states. However, the resulting spectrum still contains many properties similar to the clean limit, such as the varying sign of the supercurrent carried at different energies. This analogy holds, even though on a formal level, the calculation within our quasiclassical technique does not directly invoke these concepts.

\section{SHORT- AND LONG-JUNCTION LIMITS}

The spectrum of current-carrying states in the weak link depends very much on the ratio of the length $L$ of the weak link and the superconducting coherence length $\xi_{0}=\sqrt{D / 2 \Delta}$, or in other words, on the ratio between the superconducting order parameter $\Delta$ and the Thouless energy $E_{T}=D / L^{2}$ of the weak link. In the case of a long weak link, ${ }^{1} L \gg \xi_{0}$ (or, equivalently, $E_{T} \ll \Delta$ ), the spectrum is wide and many energy states contribute to the supercurrent with only a small phase-dependent gap of the order of a few $E_{T}$ at low energies. In the opposite limit, only the states with energy $E \in[\Delta|\cos (\phi / 2)|, \Delta]$ carry supercurrent and between these limits, $\Delta$ serves as a cutoff for the spectral supercurrent: there may exist some current-carrying states with $E>\Delta$, but their contribution vanishes quickly with $E-\Delta$.

The energy ranges can be understood as follows: In the center of the junction, which is the bottleneck for the supercurrent, both superconductors provide sufficient correlations that a gap in the energy spectrum of a size $E_{g}$ is induced at $\phi=0$, where the size of $E_{g}$ interpolates between $E_{\mathrm{T}}$ (long junction) and $\Delta$ (short junction). ${ }^{31,32}$ If now a finite phase difference is applied, the correlations from either side start to interfere more and more destructively leading to a closing of the gap at $\phi=\pi .^{33}$ Hence the lower energy bound, below which no bound states exist, is set by this phase-dependent gap. Above $\Delta$, the states depend less and less on the superconducting properties, hence their phase dependence is rapidly lost and they also do not contribute to the supercurrent.

\section{A. Short-junction limit $L \ll \xi_{0}$}

In the limit when the superconducting order parameter $\Delta$ is much smaller than the Thouless energy, the supercurrent is carried by states with energies much below $E_{T}$ and we may thus neglect the first term on the right side of Eq. (5). In this case, we get an analytical solution to the differential equations without further approximations,

$$
\begin{aligned}
& \theta(x)=\operatorname{arcosh}\left(\frac{\sqrt{\alpha^{2}+1}}{\alpha} \cosh \left[j_{E} \alpha\left(x-x_{0}\right)\right]\right), \\
& \chi(x)=\chi_{0}-\arctan \left\{\alpha \tanh \left[j_{E} \alpha\left(x-x_{0}\right)\right]\right\},
\end{aligned}
$$

where $\alpha$ and $x_{0}$ are constants which along with the spectral supercurrent $j_{E}$ are determined from the boundary conditions. In the two-probe case we can choose the origin in the center of the weak link, and assume the 
functions $\theta(x)$ and $\chi(x)$ take the bulk values at the NS boundary $(x= \pm L / 2)$. Thus we get $x_{0}=0$ and

$$
\begin{aligned}
\alpha & =\frac{\sqrt{E^{2}-\Delta^{2} \cos ^{2}(\phi / 2)}}{\Delta \cos (\phi / 2)}, \\
j_{E} & =\frac{2 \Delta \cos (\phi / 2)}{\sqrt{E^{2}-\Delta^{2} \cos ^{2}(\phi / 2)}} \operatorname{arcosh}\left(\sqrt{\frac{E^{2}-\Delta^{2} \cos ^{2}(\phi / 2)}{E^{2}-\Delta^{2}}}\right) .
\end{aligned}
$$

In the real-time calculation of the supercurrent, we are mostly interested in the imaginary part of the spectral supercurrent $j_{E}$. This is

$$
\operatorname{Im}\left(j_{E}\right)=\left\{\begin{array}{l}
0, \quad E>\Delta \\
\frac{\pi \Delta \cos (\phi / 2)}{\sqrt{E^{2}-\Delta^{2} \cos ^{2}(\phi / 2)}}, \quad E \in[\Delta|\cos (\phi / 2)|, \Delta] \\
0, \quad|E|<\Delta|\cos (\phi / 2)|,
\end{array}\right.
$$

and $\operatorname{Im}\left[j_{E}(-E)\right]=-\operatorname{Im}\left[j_{E}(E)\right]$. At $T=0$, we get the observable supercurrent by simply integrating $\operatorname{Im}\left(j_{E}\right)$ over the energy to obtain

$$
I_{S}=\frac{\pi \Delta \cos (\phi / 2)}{e R_{N}} \operatorname{artanh}[\sin (\phi / 2)]
$$

For a finite temperature, we have to multiply this by the distribution function $\tanh \left(E / 2 k_{B} T\right)$ and integrate over the energy, which is conveniently done using the Matsubara technique [i.e., substituting $E=i \omega_{n}$, where $\omega_{n}=\pi T(2 n+1)$ are the poles of $\tanh \left(E / 2 k_{B} T\right)$ and summing $\operatorname{Re}\left(j_{E}\right)$ over $n=0,1, \ldots$, see Refs. 10 and 20 for details], yielding

$$
\begin{aligned}
I_{S}= & \frac{2 \pi \Delta T}{e R_{N}} \cos (\phi / 2) \sum_{n=0}^{\infty} \frac{1}{\sqrt{\Delta^{2} \cos ^{2}(\phi / 2)+\omega_{n}^{2}}} \\
& \times \arctan \left(\frac{\Delta \sin (\phi / 2)}{\sqrt{\Delta^{2} \cos ^{2}(\phi / 2)+\omega_{n}^{2}}}\right) .
\end{aligned}
$$

As expected, Eqs. (25) and (26) are the same as obtained by Kulik and Omel'yanchuk ${ }^{34}$ and in numerical studies ${ }^{10}$ for the same limit.

In a setup where the distribution function can be controlled by an additional probe coupled to the system via a narrow normal wire (such that the current-carrying states are not essentially deformed), the resulting supercurrent as a function of control voltage $V$ at $T=0$ reads

$$
I_{S}(V)=\frac{\pi \Delta \cos (\phi / 2)}{2 e R_{N}} \ln \left[\frac{\Delta(1+\sin (\phi / 2))}{V+\sqrt{V^{2}-\Delta^{2} \cos ^{2}(\phi / 2)}}\right]
$$

for $V \in[\Delta|\cos (\phi / 2)|, \Delta]$. Above $\Delta, I_{S}$ vanishes, and for $V \leq \Delta|\cos (\phi)|$, the supercurrent has the form of Eq. (25), independent of $V$.

The spectral supercurrent of Eq. (24) can also be obtained from the diffusive limit of the corresponding quantity derived in Ref. 35. There, the supercurrent is written
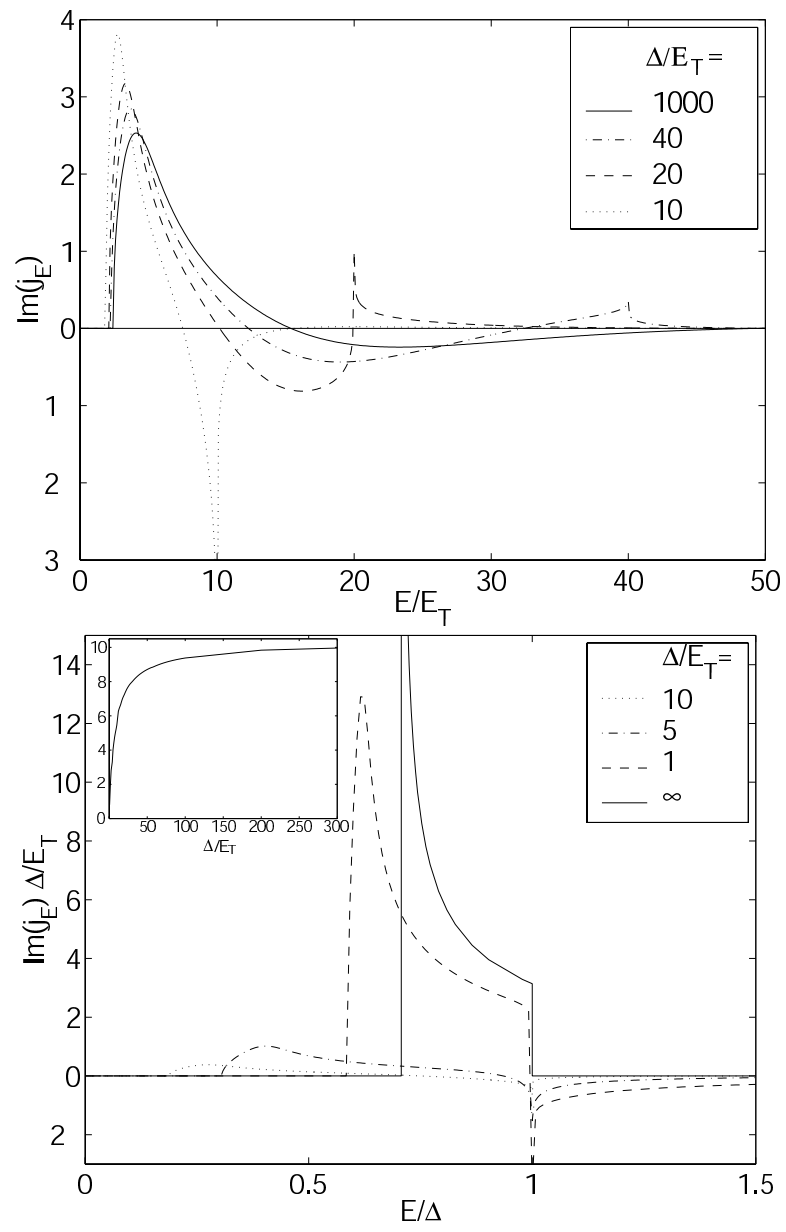

FIG. 2: Spectral supercurrent for a few values of $\Delta / E_{T}$. (a) Junctions longer than the superconducting coherence length $\xi_{0}$. A finite $\Delta / E_{T}$ shows up as a peak at $E=\Delta$. (b) Short junctions $L \sim \xi_{0}$ : the peak at $E=\Delta$ persists, but another develops around $E=\Delta \cos (\phi / 2)$. In (b), the spectral supercurrent is normalized by $E_{T} / \Delta$ to allow for the analytical solution at $E_{T} / \Delta \rightarrow \infty$. The inset shows how the zero-temperature, zero-voltage critical current behaves as a function of $\Delta / E_{T}$, in accordance with Ref. 10.

as a sum of the contributions from different bound states,

$$
I_{S}=\frac{e \Delta}{2} \sin (\phi) \sum_{p=1}^{N} \frac{\tau_{p}}{E_{p}} \tanh \left(\frac{E_{p}}{2 k_{B} T}\right),
$$

where the bound-state energies $E_{p}$ depend on the transmission eigenvalues $\tau_{p}$ by $E_{p}=\Delta\left[1-\tau_{p} \sin ^{2}(\phi / 2)\right]^{1 / 2}$. Writing Eq. (28) in the form of an energy integral,

$$
I_{S}=\frac{e \Delta}{2} \sin (\phi) \int d E \sum_{p=1}^{N} \frac{\tau_{p}}{E} \tanh \left(\frac{E}{2 k_{B} T}\right) \delta\left(E-E_{p}\right),
$$

and averaging the transmission eigenvalues over their diffusive-limit distribution, ${ }^{36} \rho(\tau)=$ $\left(\pi / 2 e^{2} R_{N}\right)(\tau \sqrt{1-\tau})^{-1}$, yields a spectral supercur- 


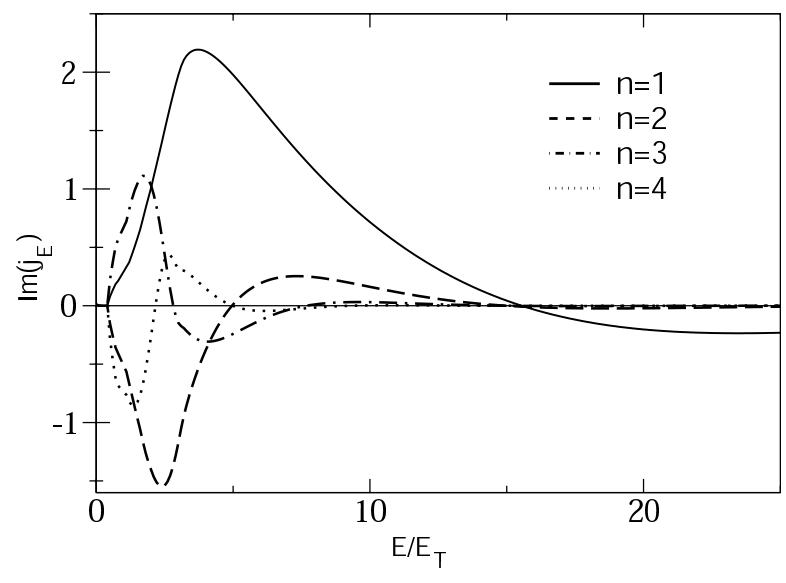

FIG. 3: Energy dependence of the Fourier sin-transformed spectral supercurrent: four lowest harmonics $n=1,2,3,4$ corresponding to the phase dependencies $\sin (n \phi)$. The energy scales and magnitudes of the different harmonic constituents decay as $1 / n$.

rent given by Eq. (24) multiplying the distribution function $\tanh \left(E / 2 k_{B} T\right)$.

\section{B. Long and intermediate-length junctions}

If the length $L$ of the weak link is much longer than $\xi_{0}$, the supercurrent is carried by a wide spectrum of energies ${ }^{1,2,37}$. At low $E$, however, the current-carrying density of states has a phase-dependent minigap reminiscent of the gap in the usual density of states of a SNS sample ${ }^{29}$. Above the gap, $\operatorname{Im}\left(j_{E}\right)$ rises sharply, then starts to oscillate with an exponentially decaying envelope. This oscillatory behavior is responsible for the occurrence of the $\pi$ state in nonequilibriumcontrolled Josephson junctions ${ }^{5,6}$ and in ferromagnetic weak links. ${ }^{38,39}$

The spectral supercurrent as a function of energy is plotted for a few values of $\Delta / E_{T}$ in Fig. 2, the upper figure showing the limit $E_{T} \ll \Delta$ and the lower the limit $E_{T} \lesssim \Delta$.

For a finite ratio $\Delta / E_{T}$, the divergence of the density of states at the superconducting gap edge is reflected as a peak in the spectral supercurrent at $E=\Delta$. The direction of the peak, positive or negative, is determined by geometric considerations and hence depends on the precise value of $\Delta / E_{T}$. For $E_{T} \approx \Delta$, the spectral supercurrent $\operatorname{Im}\left(j_{E}\right)$ tends towards the short-junction result, Eq. (24), replacing the Thouless gap by the gap of width $\Delta \cos (\phi / 2)$. Moreover, for $\Delta / E_{T} \rightarrow 0$, the width of the peak at $E=\Delta$ tends to zero.

In the limit $T \gg E_{T}$ for a long junction $\left(E_{T} \ll \Delta\right)$, the temperature dependence of the obtained observable supercurrent tends to the limits considered by Likharev ${ }^{40}$ for $\Delta \ll T$ and by Zaikin and Zharkov ${ }^{41}$ for a general $\Delta / T$.

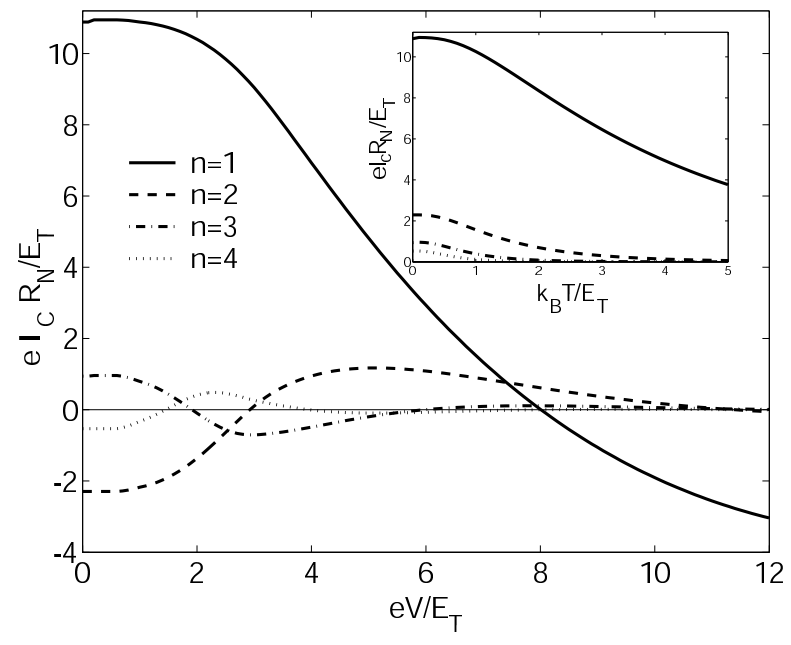

FIG. 4: Voltage dependence of the amplitudes of the first four harmonics of the observable supercurrent. Inset: Corresponding temperature dependence (even harmonics yield negative amplitudes, but here we plot the absolute values of the supercurrent).

\section{PHASE DEPENDENCE}

Originally, the Josephson effect was discovered for insulating weak links in the tunneling regime, i.e., in the limit of a very low tunneling probability. There, the supercurrent is due to an uncorrelated transfer of Cooper pairs through the weak link. ${ }^{42}$ As a result, one obtains the familiar dc Josephson relation $I_{S}=I_{c} \sin (\phi)$. However, it has been shown (see, e.g., Refs. 13 and 34) that other kinds of weak links, through which the transmission probability is much above zero, may have a different current-phase relation. Thus we may write in general

$$
I_{C}(\phi)=\sum_{n=1}^{\infty} I_{C}^{n} \sin (n \phi)
$$

where the amplitudes $I_{C}^{n}$ are the coefficients of the Fourier sine series of $I_{C}(\phi)$. For example, in a ballistic weak link where the transmission probability for Cooper pairs is $1, I_{C}^{n} \propto-(-1)^{n} / n$, yielding a sawtooth form for $I_{C}(\phi) .{ }^{13,16}$ The odd parity with respect to $\phi$ (appearance of only sine terms) of this representation reflects the fact that the supercurrent is driven by the spatial asymmetry introduced by the application of the phase: changing the phase to a negative value corresponds to mirroring the structure about the center and hence to a reversal of the current.

The occurrence of higher harmonics in Eq. (30) may be interpreted as a correlated transfer of $n$ Cooper pairs through the weak link as a result of the pairing correlations extending through quasiparticle paths containing multiple Andreev reflections. For example, the flux quantum for the $n$th harmonic is $h / 2 n e$, i.e., corresponding to a charge $2 n e$.

In a diffusive weak link considered in this paper, the 
transmission probabilities for the Cooper pairs are widely distributed between zero and $1 .{ }^{36}$ As a result, one may get contributions from the higher harmonics to the phase dependence. This is shown in Fig. 3 where the amplitudes $\operatorname{Im}\left(j_{E}^{n}\right)$ of the first four harmonics of the Fourier sine transformed spectral supercurrent through a long weak link $\left(L \gg \xi_{0}\right)$ are plotted as a function of energy. Both the amplitude of $\operatorname{Im}\left(j_{E}^{n}\right)$ and the effective energy scales decay with a power of $n$ suggesting that the observation of the higher harmonics is easiest at low temperatures. The corresponding temperature and voltage dependencies of the critical currents $I_{C}^{n}$ plotted in Fig. 4 behave analogously. A numerical fit to the obtained critical currents at $e V=k_{B} T=0$ yields roughly $I_{C}^{n} \propto-(-1)^{n} / n^{2}$ and to the voltage $V_{n}^{*}$ where $I_{C}^{n}(V)$ first changes sign suggests that the effective energy scales behave as $E_{n}^{*}=E_{T}\left(c_{1}+c_{2} / n\right)$, with some constants $c_{1,2}$.

This behavior can be understood by identifying the higher harmonics with the correlated transfer of a cluster of $n$ Cooper pairs. Now instead of the phase $\phi$, the cluster has the phase $n \phi$ and since the cycle contains $2 n$ Andreev reflections, the effective trajectory length is increased from $L$ to $n L$. In Eq. (18), making these replacements yields the observed result, $E_{n}^{*} \propto\left(c_{1}+c_{2} / n\right)$. In the diffusive limit, the effective trajectory length increases in the second power of the length of the weak link, $l_{\text {eff }} \propto L^{2}$, but since the phase is reset after each traversal through the weak link, we simply get $l_{\text {eff,n }} \propto n L^{2}$. Hence, similarly to the alternating sign, the scaling of the effective energies with index $n$ follows the behavior of the ballistic-limit spectral supercurrent.

Since the crossover voltages $V_{n}^{*}$, where $I_{C}^{n}\left(V_{n}^{*}\right)=0$, depend on $n$, the actual critical current never vanishes at the crossover: it is rather that the current-phase relation changes its form near the crossover voltages. Such a change was observed in Ref. 11, where the current-phase relation of a controllable Josephson junction was measured in a superconducting quantum interference device (SQUID) geometry.

In the short-junction regime $L \ll \xi_{0}$ the contributions of the different harmonics can be derived analytically. A general form for $\operatorname{Im}\left(j_{E}^{n}\right)$ would be complicated, but as an example, the first two amplitudes are

$$
\begin{aligned}
& \operatorname{Im}\left(j_{E}^{1}\right)=\left(\frac{E}{\Delta}\right)^{2}, \\
& \operatorname{Im}\left(j_{E}^{2}\right)=-\frac{E^{2}\left(2 \Delta^{2}-3 E^{2}\right)}{\Delta^{4}} .
\end{aligned}
$$

Clearly all the harmonics share the same energy scale, $\Delta$, but as for the case of a long weak link, the amplitude decays also here roughly as $1 / n^{2}$. Namely, integrating $\operatorname{Im}\left(j_{E}^{n}\right)$ over the energy, we get for a short weak link

$$
I_{C}^{n}=-\frac{(-1)^{n} e \Delta}{R_{N}(2 n+1)(2 n-1)} .
$$

Replacing $\Delta$ by $E_{T}$ and scaling by a numerical factor close to 33 , this form fits the amplitudes of the long-

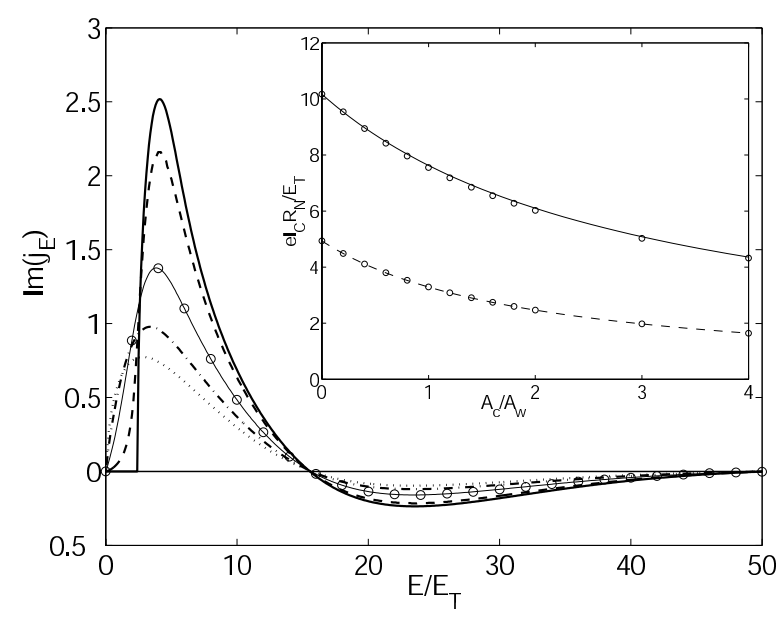

FIG. 5: Spectral supercurrent for different cross sections $A_{c}$ of the control probe. From top to bottom, the cross section is $A_{c}=0,0.2,1,2,4$ times the cross section of the weak link. Inset: observable supercurrent as a function of $A_{c}$ for $T=V=0$ (upper set of circles) and for $k_{B} T=3 E_{T}$ (lower set of circles). The zero-temperature result is fitted to $I_{S}\left(A_{c}=0\right) A_{w} /\left(A_{w}+A_{c} / 3\right)$ (solid line) and the finitetemperature result to $I_{S}\left(A_{c}=0\right) A_{w} /\left(A_{w}+A_{c} / 2\right)$ (dashed line).

junction harmonics as well but a rigorous proof does not exist.

\section{EXTRA TERMINALS}

In order to relate our results to physical observables, we have to evaluate statistical expectation values. In twoprobe SNS weak links in equilibrium, most of the experimental observations have been accurately described with the equilibrium Matsubara technique. ${ }^{10}$ However, one of the recent advances in the research of the Josephson effect has been done in nonequilibrium situations where the distribution function in the weak link has been controlled by coupling one or more normal-metal reservoirs to the weak link by phase-coherent wires. ${ }^{5,6}$ While making it possible to control the occupation of the current-carrying states, these extra wires also affect the form of $\operatorname{Im}\left(j_{E}\right){ }^{2}$ In the discussion of these effects, we concentrate on the regime of a long junction, $L \gg \xi_{0}$. There, most notably, the control probes allow for the existence of states with low energies, and therefore the Thouless gap is lifted. Moreover, the existence of the normal reservoirs brings some extra depairing by imposing a vanishing boundary condition for the pairing amplitude $f=\sinh (\theta)$ inside the reservoirs. As a result, the amplitude of the spectral supercurrent decreases due to the extra probes. In what follows, we consider the effects of the integrated cross-sectional area $A_{c}$ of the control wires attached to the center of the weak link with cross section $A_{w}$ (note: a similar effect would be present if the control wires and the weak link were made of different materials with normal- 


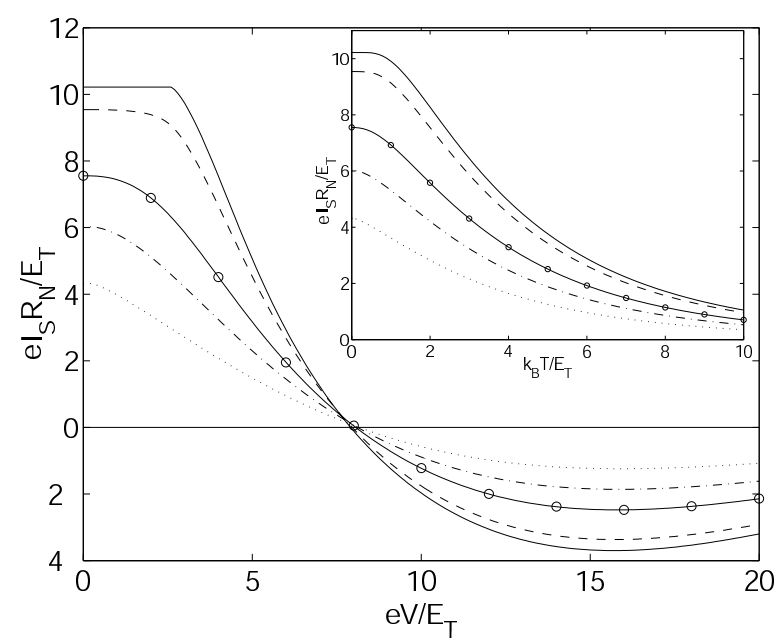

FIG. 6: Voltage dependence of supercurrent for $\phi=\pi / 2$ in the presence of the control probe with different cross sections $A_{c}$. Inset: The corresponding temperature dependence of the supercurrent. The cross sections have been chosen as in Fig. 5 .

state conductivities $\sigma_{N, c}$ and $\sigma_{N, w}$ - however, here we simply talk about $A_{c}$ and $A_{w}$ ) and of the length $L_{c}$ of the phase coherent control wires, compared to the length $L$ of the weak link. For simplicity, we assume that the widths of the control wires are much smaller than $L$, allowing one to treat these wires as quasi-1D structures, connected by the rules of Nazarov's circuit theory ${ }^{25,36}$. In the language of this circuit theory, the extra normal wires divert some of the spectral current to the normal reservoirs, thus decreasing the pairing correlations between the superconductors.

The spectral supercurrent as a function of energy for different cross sections $A_{c}$ of the control wire is plotted in Fig. 5. Here we have taken the length $L_{c}=5 L \gg L$. Already a small $A_{c} \ll A_{w}$ yields a finite $\operatorname{Im}\left(j_{E}\right)$ at low energies, but does not much reduce the total magnitude of the supercurrent. The resulting temperature and voltage dependencies of the total supercurrent $I_{s}$ is plotted in Fig. 6. Except at the very lowest voltages/temperatures of the order of the Thouless gap, the extra probes do not change the voltage/temperature dependence of $I_{s}$ from the two-probe case, but only the overall magnitude is decreased. From the resulting $I_{S}\left(A_{c}\right)$ we obtain

$$
I_{S}\left(A_{w}, A_{c}\right)=\frac{A_{w}}{A_{w}+A_{c} / 2} I_{S}\left(A_{w}, A_{c}=0\right),
$$

which holds very well for $\max \left(e V, k_{B} T\right) \gg E_{T}$.

If the length $L_{c}$ of the phase-coherent control wire is larger than $L$, the effect of the control wires is independent of the precise value of $L_{c}$. For $L_{c}<L$, the spectral supercurrent is altered for $E \lesssim \hbar D / L_{c}^{2}$ such that the overall magnitude is decreased, the observable supercurrent tending towards zero as $L_{c} \rightarrow 0$. The spectral supercurrent and the resulting temperature/voltage dependencies for $A_{c}=A_{w}$ and for three different $L_{c} / L$ are

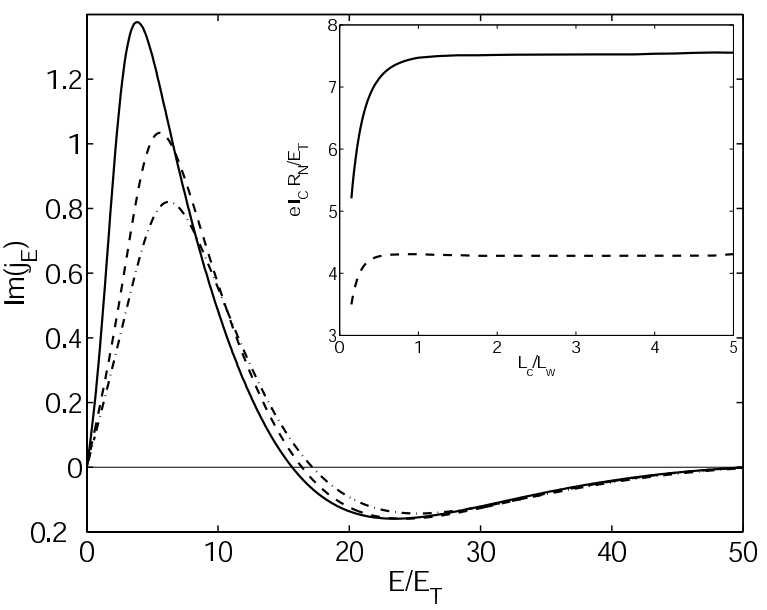

FIG. 7: Spectral supercurrent for different lengths $L_{c}$ of the control probe. From top to bottom, the length is $L_{c}=$ $5,0.25,0.15$ times the length of the weak link. The cross section of the control probe is chosen equal to that of the weak link. Inset: Supercurrent at $T=0$ (solid line) and $T=3 E_{T} / k_{B}$ (dashed) as a function of the length $L_{c}$ of the control probe with respect to the length of the weak link.

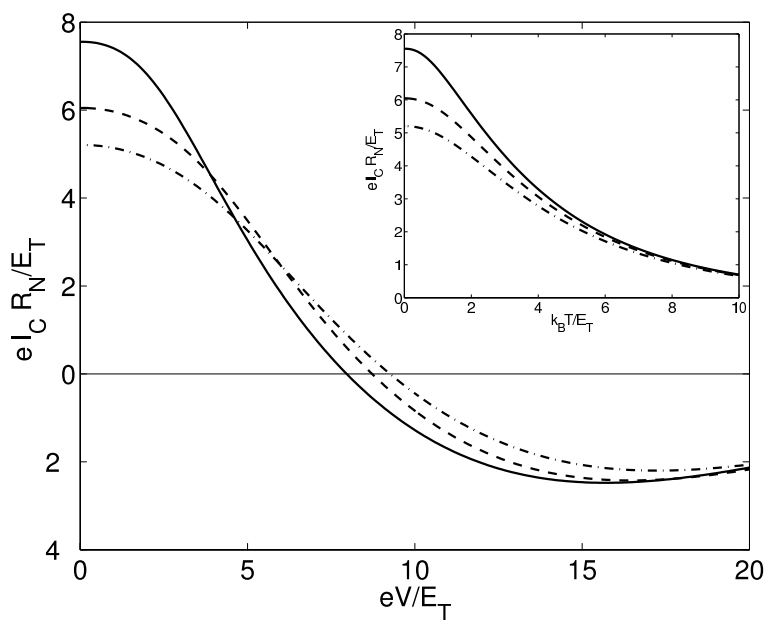

FIG. 8: Voltage dependence of supercurrent for $\phi=\pi / 2$ in the presence of a control probe with length $L_{c}$ and cross section equal to that of the weak link. Inset: The corresponding temperature dependence of the supercurrent. The lengths $L_{c}$ have been chosen as in Fig. 7 .

plotted in Figs. 7 and 8, respectively.

\section{NONIDEAL INTERFACES}

Normal-metal - superconductor interfaces with reduced transmissivity can be taken into account by specifying the transmission eigenvalues through the interface and taking them into account as in Eq. (7). Here we consider a typical case described by the distribution of eigenvalues for a "dirty" interface. 


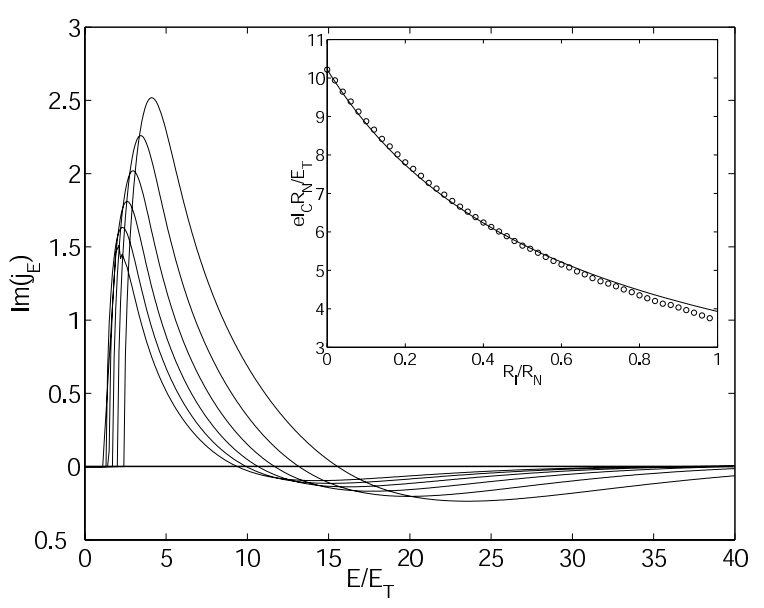

FIG. 9: Spectral supercurrent in a weak link with dirty interfaces to the superconductors, characterized by the ratio $r_{b}=R_{I} / R_{N}$ of the resistances. The interface resistance $R_{I}$ is assumed the same for both interfaces. From top to bottom: $r_{b}=0,0.2,0.4,0.6,0.8,1.0$. Enhanced scattering at the interface reduces mostly the amplitude of the supercurrent, but also slightly the effective energy scale. Inset: zero-temperature, zero-voltage supercurrent as a function of $r_{b}$. Circles: calculated supercurrent; solid line: fit to $I_{S}\left(r_{b}\right)=I_{S}\left(r_{b}=0\right) /\left(1+1.6 r_{b}\right)$.

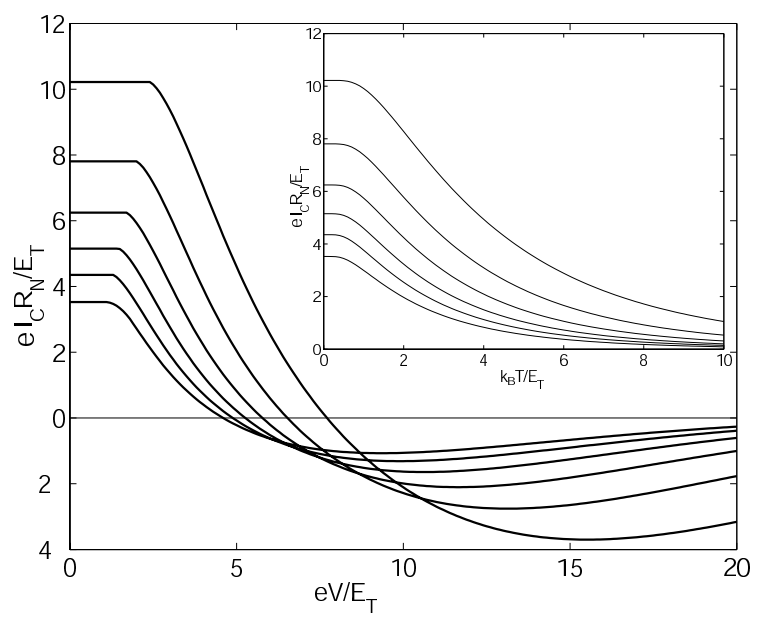

FIG. 10: Voltage dependence of the supercurrent with dirty NS interfaces at $\phi=\pi / 2$. Inset: Corresponding temperature dependence. The values of $R_{I}$ are the same as in Fig. 9.

Figure 9 shows the spectral supercurrent for a long junction connected to superconductors through a dirty interface with resistance $R_{I}$ (yielding a total resistance $R_{N}+2 R_{I}$ between the superconductors). Due to the additional resistance, the amplitude of the supercurrent decays with $r_{b}$, but also the energy scales decrease since the interface barrier can to certain extent be thought as adding a barrier-equivalent length ${ }^{43}$ to the path length of the quasiparticles. Observing the temperature and voltage dependencies of the resulting supercurrent, plotted in Fig. 10, shows that the amplitude of the supercurrent behaves at $T=0, V=0$ as

$$
I_{C}\left(R_{I}, R_{N}\right) \approx \frac{I_{C}\left(R_{I}=0\right) R_{N}}{R_{N}+1.6 R_{I}},
$$

i.e., the resistances should not simply be added up but the dirty interface decreases the supercurrent less efficiently than the normal-metal resistance. Furthermore, the effective energy scale $E^{*}$ found, e.g., from the voltage dependence indicates that it follows the approximate law $E^{*}=E_{T}\left(1+0.7 R_{I} / R_{N}\right)$. This kind of a behavior of the critical current and the spectral supercurrent is similar to those found in Ref. 24 [especially, see Eqs. (55) and (56)] and Ref. 2 in the case of a tunneling interface.

\section{CONCLUSIONS}

In this paper, we have systematically investigated the spectrum of current-carrying states in a phase coherent normal-metal weak link. Taking into account the effect of extra terminals, the characteristic energy scales - the Thouless energy $E_{T}$ and the BCS superconducting gap $\Delta$ - and a finite NS interface resistance makes it easier to find a quantitative agreement with the obtained experimental results on the nonequilibrium-controlled supercurrent. We have also been able to derive analytical results in a number of limits. Moreover, we have discussed the underlying microscopic phenomena leading to the $\pi$ state and have explained its properties, such as its dependence on energy, and higher harmonics in the phase dependence, by invoking Andreev bound states smeared over a broad distribution of times of flight, and by multiple Andreev cycles tranferring more than one Cooper pair in a single coherent process.

To obtain an optimal voltage control of the supercurrent, the interface resistances should be much smaller than the weak-link wire resistance, the control wire should be slightly longer than the weak link (but not much longer to reduce the inelastic effects on the nonequilibrium distribution function), and as thin as possible compared to the weak link. For the observation of the $\pi$ state, the ideal limit is the long-junction limit $L \gg \xi_{0}$, where the spectrum of the current-carrying states is not cut off by the superconducting gap.

In typical experiments, also inelastic scattering neglected here may become important. However, since equilibrium phenomena induced by the superconducting proximity effect have been quantitatively described by the quasiclassical theory without incorporating such effects (see, e.g., Refs. 10 and 44), we expect these inelastic effects to be mostly important in the kinetic equations describing the nonequilibrium distribution functions. In recent experiments, ${ }^{6,8}$ including these inelastic terms into the kinetic equations has lead to good agreement between the theory and the experiments. Therefore our results provide an accurate and independent way of also characterizing such inelastic effects by observing how they affect the supercurrent. 


\section{ACKNOWLEDGEMENTS}

We thank Norman Birge, Fréderic Pierre and Jochem Baselmans for discussions. This work was supported by the Graduate School in Technical Physics at the Helsinki University of Technology.
* Author to whom correspondence should be addressed. Electronic address:Tero.T.Heikkila@hut.fi; Present address: Low Temperature Laboratory, P.O. Box 2200, FIN02015 HUT, Finland

${ }^{1}$ F. K. Wilhelm, G. Schön, and A. D. Zaikin, Phys. Rev. Lett. 81, 1682 (1998).

2 S. K. Yip, Phys. Rev. B 58, 5803 (1998).

3 A. F. Volkov, Phys. Rev. Lett. 74, 4730 (1995); A. F. Volkov, R. Seviour, and V. V. Pavlovskii, Superlattices Microstruct. 25, 647 (1999).

4 A. F. Morpurgo, T. M. Klapwijk, and B. J. van Wees, Appl. Phys. Lett. 72, 966 (1998).

5 J. J. A. Baselmans, A. F. Morpurgo, B. J. van Wees, and T. M. Klapwijk, Nature (London) 397, 43 (1999); J. J. A. Baselmans, B. J. van Wees, and T. M. Klapwijk, Phys. Rev. B 63, 094504 (2001).

6 J. Huang, F. Pierre, T. T. Heikkilä, F. K. Wilhelm, and N. O. Birge, Phys. Rev. B 66, 020507(R) (2002).

7 Th. Schäpers et al., Appl. Phys. Lett. 73, 2348 (1998).

8 J. J. A. Baselmans, B. J. van Wees, and T. M. Klapwijk, Phys. Rev. B 65, 224513 (2002) .

9 J. Kutchinsky, R. Taboryski, C. B. Sørensen, J. B. Hansen, and P. E. Lindelof, Phys. Rev. Lett. 83, 4856 (1999).

10 P. Dubos, H. Courtois, B. Pannetier, F. K. Wilhelm, A. D. Zaikin, and G. Schön, Phys. Rev. B 63, 064502 (2001).

11 J. J. A. Baselmans, T. T. Heikkilä, B. J. van Wees, and T. M. Klapwijk, Phys. Rev. Lett. 89, 207002 (2002).

12 I. O. Kulik, Zh. Eksp. Teor. Fiz. 57, 1745 (1969) [Sov. Phys. JETP 30, 944 (1970)].

13 Ch. Ishii, Prog. Theor. Phys. 44, 1525 (1970).

14 J. Bardeen and J. L. Johnson, Phys. Rev. B 5, 72 (1972).

15 M. Büttiker and T. M. Klapwijk, Phys. Rev. B 33, 5114 (1986).

16 B. J. van Wees, K.-M. H. Lenssen, and C. J. P. M. Harmans, Phys. Rev. B 44, 470 (1991).

17 C. W. J. Beenakker and H. van Houten, Phys. Rev. Lett. 66, 3056 (1991).

18 U. Gunsenheimer, U. Schüssler, and R. Kümmel, Phys. Rev. B 49, 6111 (1994).

19 K. D. Usadel, Phys. Rev. Lett. 25, 507 (1970).

${ }^{20}$ For an introduction see, e.g., W. Belzig, F. K. Wilhelm, C. Bruder, G. Schön, and A. D. Zaikin, Suplatt. Microstruct. 25, 1251 (1999).

21 J. Rammer and H. Smith, Rev. Mod. Phys. 58, 323 (1986).

${ }^{22}$ F. Sols and J. Ferrer, Phys. Rev. B 49, 15913 (1994).

23 A. V. Zaitsev, Zh. Eksp. Teor. Phys. 86, 1742 (1984) [Sov. Phys. JETP 59, 1015 (1984)].

24 M. Yu. Kuprianov and V. F. Lukichev, Zh. Eksp. Teor. Fiz. 94, 139 (1988) [Sov. Phys. JETP 67, 1163 (1988)].

25 Yu. V. Nazarov, Superlattices Microstruct. 25, 1221 (1999).
${ }^{26}$ W. Belzig, A. Brataas, Yu. V. Nazarov, and G. E. W. Bauer, Phys. Rev. B 62, 9726 (2000).

27 K. M. Schep and G. E. W. Bauer, Phys. Rev. Lett. 78, 3015 (1997).

28 A. V. Zaitsev, Physica B 203, 274 (1994).

29 A. A. Golubov, F. K. Wilhelm, and A. D. Zaikin, Phys. Rev. B 55, 1123 (1997).

30 A. F. Andreev, Zh. Eksp. Teor. Fiz. 46, 1823 (1964) [Sov. Phys. JETP 19, 1228 (1964)]; 49, 655 (1965) [22, 455 (1966)].

31 A. A. Golubov and M. Yu. Kupriyanov, J. Low Temp. Phys. 70, 83 (1988).

32 W. Belzig, C. Bruder, and G. Schön, Phys. Rev. B 54, 9443 (1996).

33 F. Zhou, P. Charlat, and B. Pannetier, J. Low Temp. Phys. 110, 841 (1998).

34 I. O. Kulik and A. N. Omelyan'chuk, Fiz. Nizk. Temp. 4, 296 (1978) [Sov. J. Low Temp. Phys. 4, 142 (1978)].

35 C. W. J. Beenakker, Phys. Rev. Lett. 67, 3836 (1991); 68, 1442(E) (1992).

36 Yu. V. Nazarov, Phys. Rev. Lett. 73, 134 (1994).

37 T. T. Heikkilä, F. K. Wilhelm, and G. Schön, Europhys. Lett. 51, 434 (2000).

38 A. I. Buzdin, L. N. Bulaevskii, and V. Panyukov, Pis'ma Zh. Eksp. Teor. Fiz. 35, 147 (1982) [JETP Lett., 35, 178 (1982)]; A. I. Buzdin and M. Yu. Kupriyanov, ibid. 52, 1089 (1990) [52, 487 (1990)]; 53, 308 (1991) [53, 321 (1991)]; A. I. Buzdin, B. Bujicic, and M. Yu. Kupriyanov, Zh. Eksp. Teor. Fiz. 101, 231 (1992) [Sov. Phys. JETP, 74, 124 (1992)].

39 V. V. Ryazanov, V. A. Oboznov, A. Yu. Rusanov, A. V. Veretennikov, A. A. Golubov, and J. Aarts, Phys. Rev. Lett. 86, 2427 (2001).

${ }^{40}$ K. K. Likharev, Pis'ma Zh. Tekh. Fiz. 2, 29 (1976) [Sov. Tech. Phys. Lett. 2, 12 (1976)].

41 A. D. Zaikin and G. F. Zharkov, Fiz. Nizk. Temp. 7, 375 (1981) [Sov. J. Low Temp. Phys. 7, 184 (1981)].

42 "Uncorrelated" means in this context that no microscopic correlation between the different Cooper pairs crossing the junction exist. The transport is, however, still coherent in the sense that each Cooper pair keeps its internal correlation while traveling the junction. Furthermore, the phases of the reservoirs are assumed not to fluctuate such that the Cooper pairs are transported with the same macroscopic phase and hence add up to a macroscopic steady supercurrent.

43 F. Zhou, B. Spivak, and A. Zyuzin, Phys. Rev. B 52, 4467 (1995).

44 M. A. Sillanpää, T. T. Heikkilä, R. K. Lindell, and P. J. Hakonen, Europhys. Lett. 56, 590 (2001). 